\title{
On the doubly connected domination number of a graph
}

\author{
Joanna Cyman*, Magdalena Lemańska ${ }^{\dagger}$, Joanna Raczek ${ }^{\ddagger}$ \\ Department of Discrete Mathematics, \\ Faculty of Applied Physics and Mathematics, \\ Gdansk University of Technology, \\ Narutowicza 11/12, 80-952 Gdańsk, Poland
}

Received 3 January 2005; accepted 19 September 2005

\begin{abstract}
For a given connected graph $G=(V, E)$, a set $D \subseteq V(G)$ is a doubly connected dominating set if it is dominating and both $\langle D\rangle$ and $\langle V(G)-D\rangle$ are connected. The cardinality of the minimum doubly connected dominating set in $G$ is the doubly connected domination number. We investigate several properties of doubly connected dominating sets and give some bounds on the doubly connected domination number.

(c) Central European Science Journals Warsaw and Springer-Verlag Berlin Heidelberg. All rights reserved.
\end{abstract}

Keywords: Doubly connected domination number, connected domination number $M S C$ (2000): 05C69

\section{Introduction}

Let $G=(V, E)$ be a simple connected graph with $|V(G)|=n(G)$ and $|E(G)|=m(G)$. The neighbourhood $N_{G}(v)$ of a vertex $v$ is the set of all vertices adjacent to $v$ in $G$ and the closed neighbourhood $N_{G}[v]=N_{G}(v) \cup\{v\}$. The degree $d_{G}(v)=\left|N_{G}(v)\right|$ of a vertex $v$ is the number of edges incident to $v$ in $G$. The minimum and maximum degrees of vertices of $V(G)$ are denoted by $\delta(G)$ and $\Delta(G)$, respectively. A vertex $x$ such that $d_{G}(x)=\Delta(G)=n(G)-1$ we call a universal vertex. Let $\Omega(G)$ be the set of all endvertices of $G$, that is the set of vertices degree 1 , and let $n_{1}(G)$ be the cardinality of $\Omega(G)$.

\footnotetext{
* E-mail: joana@mif.pg.gda.pl

$\dagger$ E-mail: magda@mif.pg.gda.pl

‡ E-mail: gardenia@mif.pg.gda.pl
} 
A vertex that is a neighbour of an end-vertex is called a support. Let $S(G)$ be the set of supports in $G$.

The corona $G=H \circ K_{1}$ is the graph constructed from a copy of $H$, where for each vertex $v \in V(H)$, a new vertex $v^{\prime}$ and a pendant edge $v v^{\prime}$ are added. For disjoint graphs $G_{1}$ and $G_{2}$, the join $G=G_{1}+G_{2}$ is the graph $G$ with $V(G)=V\left(G_{1}\right) \cup V\left(G_{2}\right)$ and $E(G)=E\left(G_{1}\right) \cup E\left(G_{2}\right) \cup\left\{u v: u \in V\left(G_{1}\right) \wedge v \in V\left(G_{2}\right)\right\}$. Let us denote by $G-v$ the graph obtained from $G$ by removing the vertex $v \in V(G)$ and all edges incident to $v$.

For any connected graph $G$, a vertex $x \in V(G)$ is called a cut-vertex of $G$ if $G-x$ is no longer connected. The vertex-connectivity or simply connectivity $\kappa(G)$ is the minimum number of vertices whose removal from $G$ results in disconnected graph or a graph with only one vertex.

A set $D \subseteq V(G)$ is a dominating set of $G$ if for every vertex $v \in V(G)-D$, there exists a vertex $u \in D$ such that $v$ is adjacent to $u$. The minimum cardinality of a dominating set in $G$ is the domination number $\gamma(G)$.

Sampathkumar and Walikar [7] defined a connected dominating set $D$ to be a dominating set whose induced subgraph $\langle D\rangle$ is connected. The minimum cardinality of a connected dominating set in $G$ is the connected domination number of $G$ and is denoted by $\gamma_{c}(G)$.

In this paper we introduce a new type of domination: a set $D \subseteq V(G)$ is a doubly connected dominating set of $G$ if it is dominating and both $\langle D\rangle$ and $\langle V(G)-D\rangle$ are connected. The cardinality of a minimum doubly connected dominating set of $G$ is the doubly connected domination number of $G$ and is denoted by $\gamma_{c c}(G)$. We define that for each connected graph $G$ the set of all vertices of $G$ is a doubly connected dominating set of $G$.

For unexplained terms and symbols see $[1,5]$.

\section{Preliminary results}

We begin with some basic properties of doubly connected dominating sets.

Proposition 2.1. Let $D$ be a minimum doubly connected dominating set of a connected graph $G$ on $n \geq 3$ vertices. Then

(i) every cut-vertex is in $D$;

(ii) every support is in $D$;

(iii) at least $n_{1}(G)-1$ end-vertices are in $D$;

(iv) $\gamma_{c c}(G) \geq n_{1}(G)$, with equality if and only if $G$ is a star $K_{1, n-1}$;

(v) $\gamma_{c c}(G) \geq n_{1}(G)+|S(G)|-1$, with equality if and only if each vertex $v \in V(G)$ is either an end-vertex or a support.

Proof. (i) Assume $v$ is a cut-vertex of $G$ that does not belong to a minimum doubly connected dominating set $D$. As $G-v$ is disconnected, it is not possible to choose a connected dominating set $D \subseteq V(G)-\{v\}$, a contradiction. 
(ii) As every support is a cut-vertex, by (i) our claim follows.

(iii) If not, assume there are two end-vertices not belonging to $D$. As every support is in $D$ it follows, that $\langle V(G)-D\rangle$ is not connected, a contradiction.

(iv) By (iii), at least $n_{1}(G)-1$ end-vertices are in $D$. If $\Omega(G) \subseteq D$ our claim follows. Similarly, if there exists a vertex $x \in \Omega(G)$ such that $x \notin D$, then $\gamma_{c c}(G)=n(G)-1$ and since $n \geq 3$ we have $n(G)-1 \geq n_{1}(G)$, which completes the proof of the bound.

It is easy to see that $\gamma_{c c}\left(K_{1, n-1}\right)=n_{1}(G)$. Conversely, assume that $\gamma_{c c}(G)=$ $n_{1}(G)$. In this case, by (ii) and (iii), each support and at least all end-vertices except one are in a minimum doubly connected dominating set $D$. Thus $|S(G)|=1$, $|V(G)-D|=1$ and $|D|=n-1=n_{1}(G)$. We conclude $G$ is a star $K_{1, n-1}$.

(v) By (ii) and (iii), the inequality is straightforward. If $\Omega(G) \cup S(G)=V(G)$ then obviously $n_{1}(G)+|S(G)|-1=\gamma_{c c}(G)$. Conversely, assume that $\gamma_{c c}(G)=n_{1}(G)+$ $|S(G)|-1$. In this case, the minimum doubly connected dominating set $D$ consists of all vertices of the set $S(G)$ and all except one end-vertices. Thus $\gamma_{c c}(G)=n-1$ and $V(G)=S(G) \cup \Omega(G)$.

As an immediate consequence of Proposition 2.1 we have

Corollary 2.2. If $G=H \circ K_{1}$, then $\gamma_{c c}(G)=n(G)-1$.

Corollary 2.3. For a tree $T$ on $n \geq 3$ vertices, $\gamma_{c c}(T)=n-1$.

Proof. In a tree $T$ each vertex is either a cut-vertex or an end-vertex. By Proposition 2.1, we conclude that $\gamma_{c c}(T) \geq n-1$. On the other hand, if $x$ is an end-vertex of a tree $T$, then $D=V(T)-\{x\}$ is a doubly connected dominating set. Thus, $\gamma_{c c}(T)=n-1$.

Since every doubly connected dominating set is a connected dominating and every connected dominating set is dominating, we have the following inequality chain for every connected graph $G$ :

$$
\gamma(G) \leq \gamma_{c}(G) \leq \gamma_{c c}(G)
$$

We characterize now some graphs for which the numbers $\gamma_{c c}(G)$ and $\gamma_{c}(G)$ are the same.

Proposition 2.4. Let $G$ be a connected graph on $n \geq 3$ vertices.

(i) If $G$ is a cycle, then $\gamma_{c c}(G)=\gamma_{c}(G)=n-2$.

(ii) If $\gamma_{c c}(G)=\gamma_{c}(G)$, then $\gamma_{c c}(G) \leq n-2$.

(iii) If $\gamma_{c c}(G)=\gamma_{c}(G)$, then $\delta(G) \geq 2$.

(iv) For any unicyclic graph $G$ we have $\gamma_{c}(G)=\gamma_{c c}(G)$ if and only if $G$ is a cycle.

Proof. (i) It is obvious.

(ii) It is known [7] that for every connected graph $G$ with $n \geq 3$ we have $\gamma_{c}(G) \leq n-2$. Thus, for the equality $\gamma_{c c}(G)=\gamma_{c}(G)$ we conclude that $\gamma_{c c}(G) \leq n-2$.

(iii) Suppose $\gamma_{c c}(G)=\gamma_{c}(G)$ and $x$ is an end-vertex in $G$. By (ii), $\gamma_{c c}(G) \leq n-2$. Let $D$ 
be a minimum doubly connected dominating set of $G$ of cardinality $|D| \leq n-2$. If $x \in D$, then $D-\{x\}$ is also a connected dominating set of $G$, a contradiction with equality $\gamma_{c c}(G)=\gamma_{c}(G)$. If $x \notin D$, then $x$ is the unique vertex in $V(G)-D$, because $\langle V(G)-D\rangle$ is connected. Thus $\gamma_{c c}(G)=n-1$, a contradiction.

(iv) If $G$ is a cycle on $n$ vertices, then by (i) $\gamma_{c c}(G)=\gamma_{c}(G)=n-2$. Suppose now $G$ is unicyclic with $\gamma_{c}(G)=\gamma_{c c}(G)$ and $G$ is not a cycle. By (ii), $\gamma_{c c}(G) \leq n-2$. Moreover, there exists a vertex $x \in V(G)$ such that $d_{G}(x)=1$. Let $D$ be a minimum doubly connected dominating set of $G$. If $x \notin D$, then $\gamma_{c c}(G)=n-1$, a contradiction. On the other hand, $x \in D$ implies, that $D-\{x\}$ is a connected dominating set of $G$, a contradiction, as $\gamma_{c}(G)=\gamma_{c c}(G)$.

We have shown that there exist graphs $G$ for which the equality $\gamma_{c}(G)=\gamma_{c c}(G)$ holds. However the difference between $\gamma_{c c}(G)$ and $\gamma_{c}(G)$ can be arbitrarily large.

Lemma 2.5. The difference $\gamma_{c c}-\gamma_{c}$ can be arbitrarily large.

Proof. Consider a star $K_{1, n-1}$ with $n-1$ end-vertices. Of course, $\gamma_{c}\left(K_{1, n-1}\right)=1$. By Proposition 2.1, $\gamma_{c c}\left(K_{1, n-1}\right)=n-1$. Thus $\gamma_{c c}\left(K_{1, n-1}\right)-\gamma_{c}\left(K_{1, n-1}\right)=n-2$.

Observation 2.6. Let $G=K_{m_{1}, m_{2}, \ldots, m_{k}}$ be the complete $k$ partite graph, $k \geq 3$ with $m_{1} \leq m_{2} \leq \cdots \leq m_{k}$.

- If $m_{1}=1$, then $\gamma_{c c}(G)=1$;

- If $m_{1} \geq 2$, then $\gamma_{c c}(G)=2$.

Observation 2.7. If $G_{1}$ and $G_{2}$ are disjoint connected graphs, then

$$
\gamma_{c c}\left(G_{1}+G_{2}\right)= \begin{cases}1 & \text { if } \gamma_{c c}\left(G_{1}\right)=1 \text { or } \gamma_{c c}\left(G_{2}\right)=1 \\ 2 & \text { otherwise }\end{cases}
$$

A connected subgraph $B$ of $G$ is called $a$ block if $B$ has no cut-vertex and every subgraph $B^{\prime} \subseteq G$ with $B \subseteq B^{\prime}$ and $B \neq B^{\prime}$ has at least one cut-vertex. A connected graph $G$ is called a block graph if every block in $G$ is complete. A vertex $v$ of a graph $G$ is called a simplicial vertex if every two vertices of $N_{G}(v)$ are adjacent in $G$.

Theorem 2.8. If $G$ is a block graph, then $\gamma_{c c}(G)=n(G)-t$, where $t$ is the maximal number of simplicial vertices in a block with a largest number of simplicial vertices.

Proof. Let $D$ be a minimum doubly connected dominating set of a block graph $G$. By Proposition 2.1, each cut-vertex belongs to $D$. Hence $\gamma_{c c}(G) \geq n(G)-t$, where $t$ is maximal number of simplicial vertices in a block with a largest number of simplicial vertices.

Conversely, let $B$ be a block with a largest number of simplicial vertices. Denote by $F$ the set of all simplicial vertices belonging to $B$ and let $|F|=t$. Then $V(G)-F$ is a doubly 
connected dominating set of $G$ and we have $\gamma_{c c}(G) \leq n(G)-t$. Thus $\gamma_{c c}(G)=n(G)-t$.

\section{Bounds}

Now we find some bounds on the doubly connected domination number. For this purpose, denote by $\mathcal{A}$ a family of graphs such that $K_{2} \in \mathcal{A}$ and $G$ belongs to $\mathcal{A}$ if and only if for each pair of adjacent non-cut-vertices $u, v \in V(G),\langle V(G)-\{u, v\}\rangle$ is disconnected.

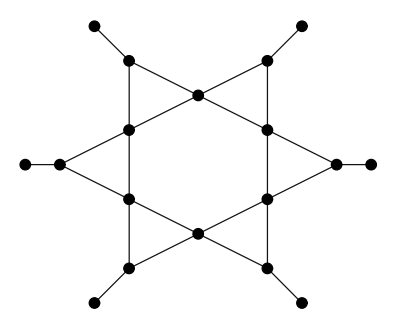

Fig. 1 A graph $G \in \mathcal{A}$.

Theorem 3.1. For every connected graph $G$ on $n \geq 2$ vertices,

$$
1 \leq \gamma_{c c}(G) \leq n-1
$$

with equality for the lower bound if and only if there exists a connected graph $H$ such that $G=H+K_{1}$ and equality for the upper bound if and only if $G \in \mathcal{A}$.

Proof. The inequality $1 \leq \gamma_{c c}(G)$ is obvious. If $G=H+K_{1}$ and $H$ is connected, then obviously $\gamma_{c c}(G)=1$. Assume now that $\gamma_{c c}(G)=1$ and let $D=\{x\}$ be a minimum doubly connected dominating set of $G$. Since $D$ is dominating, $x$ must be a universal vertex. Moreover, $\langle V(G)-D\rangle=\langle V(G)-\{x\}\rangle$ is connected, so $x$ is a non-cut-vertex. We conclude that $G=H+K_{1}$, where $H$ is connected.

Now we prove that $\gamma_{c c}(G) \leq n-1$. The inequality and the equality are straightforward when $G=K_{2}$. Suppose $n \geq 3$. Then there exist in $G$ at least two non-cut-vertices, for example two leaves of a spanning tree of $G$. Let $x$ be a non-cut-vertex. Then $D=$ $V(G)-\{x\}$ is a doubly connected dominating set of $G$.

If $G \in \mathcal{A}$, then $\gamma_{c c}(G)=n-1$, because every support of $G$ is in $D$ and for each pair of adjacent non-cut-vertices $u, v \in V(G)$, the induced subgraph $\langle V(G)-\{u, v\}\rangle$ is disconnected. Now let $G \notin \mathcal{A}$. It suffices to show that $\gamma_{c c}(G) \leq n-2$. If $G \notin \mathcal{A}$, then there exist adjacent non-cut-vertices $v, u \in V(G)$ such that $\langle V(G)-\{u, v\}\rangle$ is connected. In this case $D=V(G)-\{u, v\}$ is a doubly connected dominating set of $G$, as $n \geq 3, G$ is connected and neither of $u, v$ is a support.

Proposition 3.2. Let $G$ be a connected graph on $n \geq 2$ vertices. Then $\gamma_{c c}(G) \leq n-$ $\kappa(G)+1$. 
Proof. If $\kappa(G) \leq 2$, then by Theorem 3.1 our claim follows. Thus assume now $\kappa(G) \geq 3$. It is obvious that $\kappa(G) \leq \delta(G)$. Let $A$ be a set of an arbitrary vertex $x \in V(G)$ and $\kappa(G)-2$ of its neighbours. Obviously, $\langle V(G)-A\rangle$ is connected. Observe that $D=$ $V(G)-A$ is dominating in $G$. Thus $D$ is a doubly connected dominating set in $G$ with $|D|=n-\kappa(G)+1$.

In [7] Sampathkumar and Walikar showed that for every connected graph $G$ with $n \geq 3$ vertices and $m$ edges we have inequalities $\frac{n}{\Delta(G)+1} \leq \gamma_{c}(G) \leq 2 m-n$. Now we present similar inequalities for the number $\gamma_{c c}$.

Theorem 3.3. For any connected graph $G$ with $n \geq 2$ vertices and $m$ edges,

$$
\frac{n}{\Delta(G)+1} \leq \gamma_{c c}(G) \leq 2 m-n+1
$$

with equality for the lower bound if and only if $\gamma_{c c}(G)=1$ and equality for the upper bound if and only if $G$ is a tree.

Proof. Since $\frac{n}{\Delta(G)+1} \leq \gamma_{c}(G) \leq \gamma_{c c}(G)$ the lower bound follows. If $\gamma_{c c}(G)=1$, then by Theorem 3.1 there exists a vertex $v \in V(G)$ such that $d_{G}(v)=n-1$. Thus $\frac{n}{\Delta(G)+1}=1=$ $\gamma_{c c}(G)$.

Conversely, let $G$ be a graph such that $\gamma_{c c}(G)=\frac{n}{\Delta(G)+1}$ and $\gamma_{c c}(G)>1$. Let $D$ be a minimum doubly connected dominating set of $G$. Since $\langle D\rangle$ is connected, for each $v \in D$ we have $\left|N_{G}(v) \cap(V(G)-D)\right| \leq \Delta(G)-1$. Hence $|V(G)-D| \leq(\Delta(G)-1)|D|$ and $n-\gamma_{c c}(G) \leq(\Delta(G)-1) \gamma_{c c}(G)$, which gives $\gamma_{c c}(G) \geq \frac{n}{\Delta(G)}$, a contradiction.

By Theorem 3.1, $\gamma_{c c}(G) \leq n-1=2(n-1)-n+1$ and since $G$ is connected, $m \geq n-1$. Thus $\gamma_{c c}(G) \leq 2 m-n+1$.

We now show that $\gamma_{c c}(G)=2 m-n+1$ if and only if $G$ is a tree. If $G$ is a tree, then $m=n-1$ and $\gamma_{c c}(G)=n-1=2 m-n+1$. Conversely, let $\gamma_{c c}(G)=2 m-n+1$. By Theorem 3.1 we have $2 m-n+1 \leq n-1$, which implies $m \leq n-1$, so $G$ must be a tree with $m=n-1$.

As an immediate consequence of the second paragraph of the proof of Theorem 3.3 we have what follows.

Corollary 3.4. For each connected graph $G$ with $\gamma_{c c}(G)>1$ is $\gamma_{c c}(G) \geq \frac{n}{\Delta(G)}$.

Now we introduce the following notation: if $T_{1}$ and $T_{2}$ are vertex disjoint trees, then by $\mathcal{P}\left(T_{1}, T_{2}\right)$ we denote the set of all graphs $G$ that can be obtained from $T_{1}$ and $T_{2}$ by adding $n\left(T_{2}\right)$ edges, one edge joining each vertex of $T_{2}$ to one arbitrarily chosen vertex of $T_{1}$. We say that a graph $G$ belongs to the family $\mathcal{U}$ if there exist trees $T_{1}$ and $T_{2}$ such that $G \in \mathcal{P}\left(T_{1}, T_{2}\right)$.

Theorem 3.5. For any connected graph $G$ on $n \geq 2$ vertices and with $m$ edges,

$$
2 n-m-2 \leq \gamma_{c c}(G)
$$




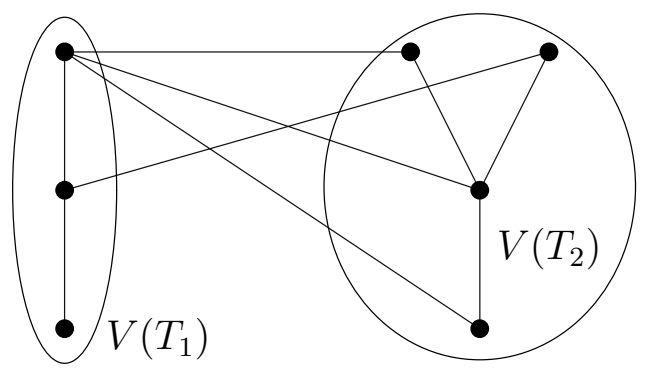

Fig. 2 A graph $G \in \mathcal{P}\left(T_{1}, T_{2}\right)$.

with equality for the bound if and only if $G$ belongs to the family $\mathcal{U}$.

Proof. Let $D$ be a minimum doubly connected dominating set in $G$. Since $\langle D\rangle$ and $\langle V(G)-D\rangle$ are connected and $D$ is dominating, we have the following inequalities:

$$
\begin{aligned}
m(\langle D\rangle) & \geq \gamma_{c c}(G)-1, \\
m(\langle V(G)-D\rangle) & \geq n-\gamma_{c c}(G)-1, \\
m_{\gamma_{c c}} & \geq n-\gamma_{c c}(G),
\end{aligned}
$$

where $m_{\gamma_{c c}}$ is the number of the edges connecting vertices of $V(G)-D$ to vertices of $D$. By summing the inequalities we obtain

$$
m=m(\langle D\rangle)+m(\langle V-D\rangle)+m_{\gamma_{c c}} \geq 2 n-\gamma_{c c}(G)-2
$$

and thus $2 n-m-2 \leq \gamma_{c c}(G)$.

We now show that $\gamma_{c c}(G)=2 n-m-2$ if and only if $G$ belongs to the family $\mathcal{U}$. Let $G \in \mathcal{U}$. Then there exist trees $T_{1}$ and $T_{2}$ such that $G \in \mathcal{P}\left(T_{1}, T_{2}\right)$. In such a graph $G$ the set $V\left(T_{1}\right)$ is a doubly connected dominating set. Thus $\gamma_{c c}(G) \leq n\left(T_{1}\right)$. Of course $n=n\left(T_{1}\right)+n\left(T_{2}\right)$ and

$$
m=m\left(T_{1}\right)+m\left(T_{2}\right)+n\left(T_{2}\right)=n\left(T_{1}\right)-1+n\left(T_{2}\right)-1+n\left(T_{2}\right)=n\left(T_{1}\right)+2 n\left(T_{2}\right)-2 .
$$

It follows that

$$
2 n-m-2=2\left(n\left(T_{1}\right)+n\left(T_{2}\right)\right)-\left(n\left(T_{1}\right)+2 n\left(T_{2}\right)-2\right)-2=n\left(T_{1}\right) .
$$

Consequently $\gamma_{c c}(G) \geq n\left(T_{1}\right)$, which together with $\gamma_{c c}(G) \leq n\left(T_{1}\right)$ gives $\gamma_{c c}(G)=n\left(T_{1}\right)=$ $2 n-m-2$.

Conversely, suppose $\gamma_{c c}(G)=2 n-m-2$. This implies that

$$
\begin{aligned}
m(\langle D\rangle) & =\gamma_{c c}(G)-1=n(\langle D\rangle)-1, \\
m(\langle V(G)-D\rangle) & =n-\gamma_{c c}(G)-1=n(\langle V(G)-D\rangle)-1, \\
m_{\gamma_{c c}} & =n-\gamma_{c c}(G) .
\end{aligned}
$$

It follows that $\langle D\rangle$ and $\langle V(G)-D\rangle$ are trees and each vertex of $V(G)-D$ has exactly one neighbour in $D$. Thus $G$ is a graph obtained from two trees $T_{1}$ and $T_{2}$ by adding $n\left(T_{2}\right)$ edges, one edge joining each vertex of $T_{2}$ to one arbitrarily chosen vertex of $T_{1}$. 
Duchet and Meyniel [3] have shown that for any connected graph $G$ is $\gamma_{c}(G) \leq$ $2 \beta_{0}(G)-1$ and $\gamma_{c}(G) \leq 2 \Gamma(G)-1$, where $\Gamma(G)$ is the maximum cardinality of a minimal dominating set of $G$ and $\beta_{0}$ is the maximum cardinality of an independent set of $G$. The next theorem shows that there is no similar result for the doubly connected domination number of a graph.

Theorem 3.6. Each of the differences $\gamma_{c c}-\beta_{0}$ and $\gamma_{c c}-\Gamma$ can be arbitrarily large.

Proof. We show a graph $G$ for which $\gamma_{c c}(G)-\beta_{0}(G)=\gamma_{c c}(G)-\Gamma(G)=k$ for any positive integer $k$. Let $G$ be a corona $K_{k+1} \circ K_{1}$. It is easy to observe that the set of end-vertices $\Omega(G)$ is the maximum independent set of $G$ and thus $\beta_{0}(G)=k+1$. The set $\Omega(G)$ is also the maximum minimal dominating set of $G$, so $\Gamma(G)=k+1$. Since $G$ is a corona, from Corollary 2.2 we have $\gamma_{c c}(G)=|V(G)|-1=2(k+1)-1=2 k+1$. It follows that $\gamma_{c c}(G)-\beta_{0}(G)=\gamma_{c c}(G)-\Gamma(G)=k$.

\section{Edge subdivision and vertex removing}

Now we examine the effects on $\gamma_{c c}(G)$ when $G$ is modified by an edge subdivision.

An edge subdivision in a nonempty graph $G$ is an operation of removal of an edge $e=u v$ and the addition of a new vertex $w$ and edges $u w$ and $v w$. A graph obtained from $G$ by subdividing the edge $e=u v$ is denoted by $G \oplus w_{u v}$.

Theorem 4.1. For every connected graph $G$ we have $\gamma_{c c}(G) \leq \gamma_{c c}\left(G \oplus w_{u v}\right)$.

Proof. Let $e=u v$ be the subdivided edge and let $D_{0}$ be a minimum doubly connected dominating set of $G \oplus w_{u v}$. We consider two cases:

a) $w \in D_{0}$. Then, since $\left\langle D_{0}\right\rangle$ is connected, $u$ or $v$ belong to $D_{0}$. If both of these vertices belong to $D_{0}$, then $D_{0}-\{w\}$ is a doubly connected dominating set of $G$ and thus $\gamma_{c c}(G)<\left|D_{0}\right|=\gamma_{c c}\left(G \oplus w_{u v}\right)$. If $u \in D_{0}$ and $v \notin D_{0}$, then $D_{0}-\{w\}$ is a doubly connected dominating set of $G$ and we have the required inequality.

b) $w \notin D_{0}$. Then, since $D_{0}$ is dominating, $u$ or $v$ belong $D_{0}$. Then, similarly as in case $a)$, we have $\gamma_{c c}(G) \leq\left|D_{0}\right|=\gamma_{c c}\left(G \oplus w_{u v}\right)$.

Theorem 4.2. The difference $\gamma_{c c}\left(G \oplus w_{u v}\right)-\gamma_{c c}(G)$ can be arbitrarily large.

Proof. We construct graphs $G$ and $G \oplus w_{u v}$ for which $\gamma_{c c}\left(G \oplus w_{u v}\right)-\gamma_{c c}(G)=k$ for a non-negative integer $k \geq 2$.

We begin with two stars $K_{1, k-1}, k \geq 2$ and denote their centers by $u$ and $v$. Next we add a vertex $x$ and edges joining $x$ with all vertices of the stars. Finally, to obtain a graph $G$, we add an edge $e=u v$ and a pendant edge $x x^{\prime}$ (see Fig. 3). It is easy to observe that the set $D=\left\{x, x^{\prime}\right\}$ is a minimum doubly connected dominating set of $G$ and thus $\gamma_{c c}(G)=2$. 
For the graph $G \oplus w_{u v}$ notice that the set $D_{u}=N[v] \cup\left\{x^{\prime}\right\}-\{w\}$ is a minimum doubly connected dominating set and the size of this set is $k+2$. Thus $\gamma_{c c}\left(G \oplus w_{u v}\right)-\gamma_{c c}(G)=$ $k+2-2=k$.
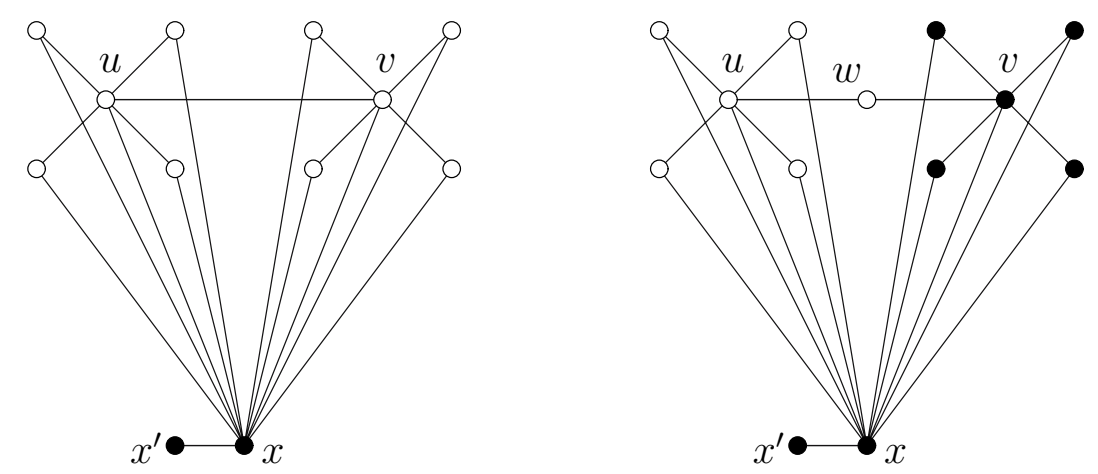

Fig. 3 Graphs $G$ and $G \oplus w_{u v}$ for $k=5$.

Theorem 4.3. The difference $\gamma_{c c}(G)-\gamma_{c c}(G-x)$ can be arbitrarily large.

Proof. Let $H$ be the join $K_{1, k}+K_{1}, k \geq 2$, and let $G$ be the graph that results if we add two pendant edges and two end-vertices $x$ and $y$ to the vertices of degree $k+1$ of the graph $H$ (see Fig. 4). It is easy to observe that $V(G)-\{x\}$ is a minimum doubly connected dominating set of $G$. Thus, $\gamma_{c c}(G)=k+3$.

The set $N_{G}[y]$ is a minimum doubly connected dominating set of $G-x$. Thus $\gamma_{c c}(G-$ $x)=2$ and finally we have $\gamma_{c c}(G)-\gamma_{c c}(G-x)=k+1$.
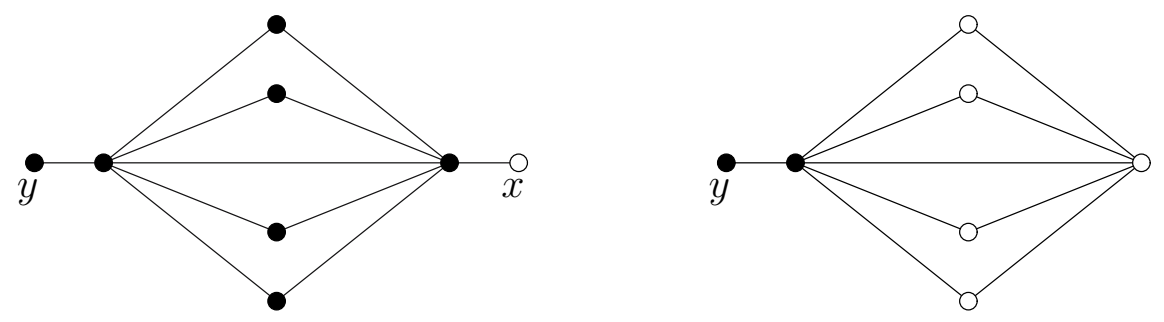

Fig. 4 Graphs $G$ and $G-x$ for $k=4$.

Theorem 4.4. The difference $\gamma_{c c}(G-x)-\gamma_{c c}(G)$ can be arbitrarily large.

Proof. Let $G$ be the join of a path $P$ on $n$ vertices and $K_{1}$. Let $x$ be the vertex of $K_{1}$. Clearly we have $\gamma_{c c}(G)=1$.

As $G-x$ is a tree, by Corollary 2.3 we have $\gamma_{c c}(G-x)=n-1$. Thus $\gamma_{c c}(G-x)-$ $\gamma_{c c}(G)=n-2$. 


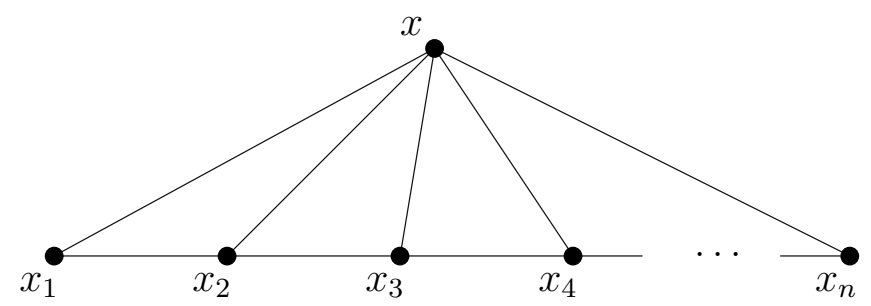

Fig. 5 Graph $G$.

\section{Complexity issues for $\gamma_{c c}$}

In this section we consider the decision problem of DOUBLY CONNECTED DOMINATING SET as follows

\section{DOUBLY CONNECTED DOMINATING SET (DCDS)}

INSTANCE: A connected graph $G=(V, E)$ and a positive integer $k$.

QUESTION: Does $G$ have a doubly connected dominating set of size at most $k$ ?

We show that the decision problem DCDS is NP-complete, even when restricted to connected bipartite graphs. We will use a well-known NP-completeness result, called DOMINATING SET, which is defined as follows.

\section{DOMINATING SET (DS)}

INSTANCE: A graph $G=(V, E)$ and a positive integer $k$.

QUESTION: Does $G$ have a dominating set of size at most $k$ ?

Garey and Johnson in [4] proved that DS is $\mathrm{NP}-$ complete.

Theorem 5.1. DCDS for bipartite graphs is NP-complete.

Proof. We know that DCDS problem for bipartite graphs is in class NP of decision problems as it is easy to verify in polynomial time whether $D$ is a doubly connected dominating set.

For any given instance for DS, which is a graph $G=(V, E)$ and an integer $k$, we construct a graph $H$ and an integer $q$ as follows: 


$$
\begin{aligned}
V(H) & =V(G) \times\{1,2,3\} \cup\{x, y\}, \\
E(H) & =\{(v, 1)(v, 2): v \in V(G)\} \\
& \cup\{(v, 2)(v, 3): v \in V(G)\} \\
& \cup\{(v, 1) x: v \in V(G)\} \\
& \cup\{(v, 1) y: v \in V(G)\} \\
& \cup\{(v, 3) x: v \in V(G)\} \\
& \cup\{(v, 3) y: v \in V(G)\} \\
& \cup\{(v, 1)(w, 2): v w \in E(G)\}, \\
q & =k+1 .
\end{aligned}
$$

The graph $H$ is connected and bipartite, as every cycle in $H$ has even length. (See Figure $6)$.

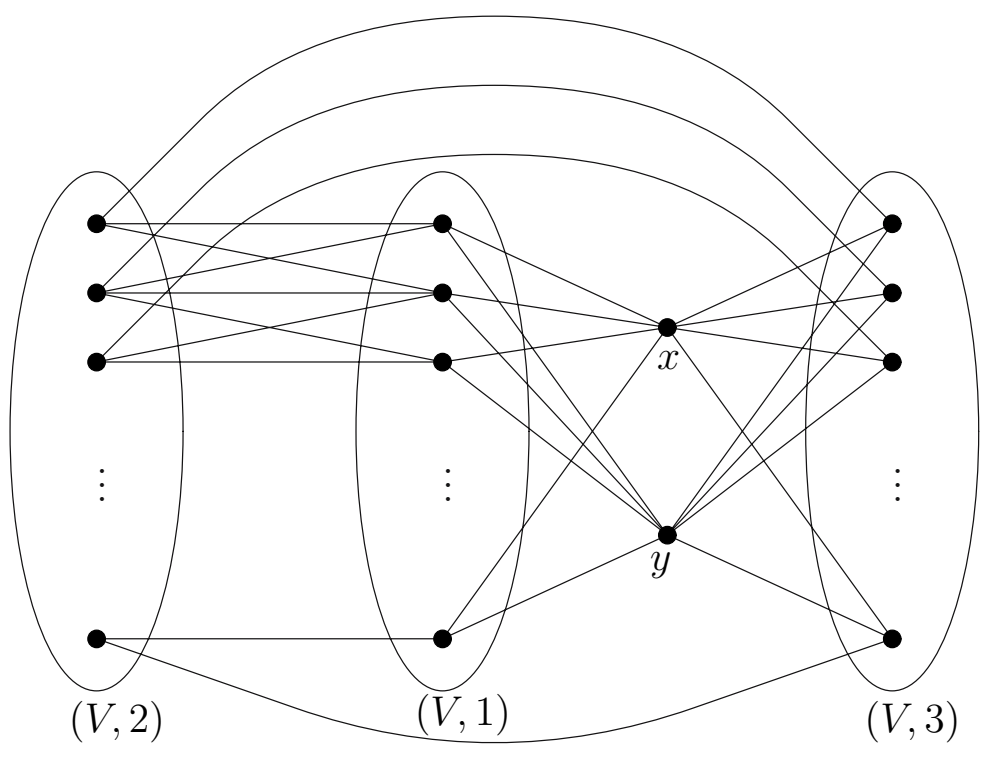

Fig. 6 Reduction from DS to DCDS for bipartite graphs.

Assume first that $G$ has a dominating set $D=\left\{v_{1}, v_{2}, \ldots, v_{k^{\prime}}\right\}, k^{\prime} \leq k$, of size at most $k$. Let $F=\left\{\left(v_{1}, 1\right),\left(v_{2}, 1\right), \ldots,\left(v_{k^{\prime}}, 1\right), x\right\}$. Since $x$ dominates all vertices in $(V, 1) \cup(V, 3)$ and $D$ is a dominating set in $G$, the set $F$ is dominating in $H$. Moreover, from the construction of $H$ we see that induced subgraphs $\langle F\rangle$ and $\langle V(H)-F\rangle$ are connected. Thus $F$ is a doubly connected dominating set of $H$ of size at most $q=k+1$.

Conversely, assume that $F$ is a doubly connected dominating set of cardinality at most $q$ in $H$. We shall show that $G$ contains a dominating set $D$ of size at most $k=q-1$. It is easy to see that if $q>n(G)$, answers for problems DCDS and DS are "yes". So assume $q \leq n(G)$. We claim that either vertex $x$ or $y$ is in every doubly connected dominating set of size $q \leq n(G)$, because a connected dominating set of size at most $n(G)$ that dominates all vertices of $(V, 3)$ and does not contain $x$ nor $y$ does not exist. (Observe 
that in $\langle V \times\{1,2,3\}\rangle$ the subset $(V, 3)$ is a set of vertices of degree 1.) Thus assume $x \in F$. Moreover, every doubly connected dominating set $F^{\prime}$ of size $q_{1} \leq n(G)$ can be transformed into a doubly connected dominating set $F \subseteq(V, 1) \cup\{x\}$ of size $q \leq q_{1}$ as follows

- $x \in F$;

- if $\left(v_{i}, 1\right) \in F^{\prime}$, then $\left(v_{i}, 1\right) \in F$;

- if $\left(v_{i}, 3\right) \in F^{\prime}$, then $\left(v_{i}, 1\right) \in F$;

- if $\left(v_{i}, 2\right) \in F^{\prime}$, then $\left(v_{i}, 1\right) \in F$.

Now, if $F=\left\{\left(v_{1}, 1\right),\left(v_{2}, 1\right), \ldots,\left(v_{q-1}, 1\right), x\right\}$ is a doubly connected dominating set of size $q$, then $D=\left\{v_{1}, v_{2}, \ldots, v_{q-1}\right\}$ is a dominating set in $G$ of size $k=q-1$.

It is obvious that the transformation used is polynomial, as $H$ has $3 n(G)+2$ vertices and $4 n(G)+2 m(G)$ edges.

\section{References}

[1] J.A. Bondy and U.S.R. Murty: Graph Theory with Applications, Macmillan, London, 1976.

[2] C. Bo and B. Liu: "Some inequalities about connected domination number", Disc. Math., Vol. 159, (1996), pp. 241-245.

[3] P. Duchet and H. Meyniel: "On Hadwiger's number and the stability number", Ann. Disc. Math., Vol. 13, (1982), pp. 71-74.

[4] M.R. Garey and D.S. Johnson: Computers and Intractability: A Guide to the Theory of NP-completeness, Freeman, San Francisco, 1979.

[5] T.W. Haynes, S.T. Hedetniemi and P.J. Slater: Fundamentals of Domination in Graphs, Marcel Dekker, New York, 1998.

[6] S.T. Hedetniemi and R. Laskar: Connected domination in graphs, Graph Theory and Combinatorics, Academic Press, London, 1984, pp. 209-217.

[7] E. Sampathkumar and H.B. Walikar: "The connected domination number of a graph", J. Math. Phys. Sci., Vol. 13, (1979), pp. 607-613. 